\title{
OPTIMAL PILOT TONES FOR MIMO INTERLEAVED OFDM SYSTEMS
}

\author{
Vinod T. S. and K. V. S. Hari \\ ECE Department, IISc, Bangalore - 560012, INDIA. \\ vinodts@protocol.ece.iisc.ernet.in, hari@ece.iisc.ernet.in
}

\begin{abstract}
This paper describes a channel estimation scheme for MIMO Interleaved Orthogonal Frequency Division Multiplexing(IOFDM) systems. The mean square error(MSE) of the proposed scheme is derived, and for the MSE of the channel estimates to be minimized, it is shown in this paper that the pilot tones will have to be equispaced in frequency, equipowered, with the entire power being allotted to the pilot tones in one of the $P$ data blocks of an IOFDM block, and the tones in the corresponding positions in the remaining $P-1$ data blocks being equal to zero. Through simulations, a comparison is made between the proposed training scheme for MIMO-IOFDM and other training sequences, and also between the proposed training scheme and the existing schemes which estimate the channel over $P$ consecutive blocks.
\end{abstract}

\section{INTRODUCTION}

High data rate techniques in communication systems have gained considerable attraction in recent years. A strong candidate for these systems is Orthogonal Frequency Division Multiplexing (OFDM) which is a multi-carrier modulation technique. Conventional OFDM systems transform information symbol blocks and then insert redundancy in the form of either cyclic prefix $(\mathrm{CP})$ or zero padding(ZP) in order to avoid inter-block interference(IBI). To minimize the reduction in code rate due to $\mathrm{CP} / \mathrm{ZP}$ in OFDM systems, an OFDM variate, called the IOFDM system was proposed in [1], which performs well when the channel is varying slowly.

In conditions where the channel does not vary over a few OFDM blocks, the design of pilot tones for MIMOOFDM systems, with the channel being estimated over multiple OFDM blocks, has been presented in [2]. Channel estimation issues for IOFDM have not been dealt with in [1] or in the STBC scheme proposed in [4]. In this paper, optimal pilot tones are designed for the generic case of MIMOIOFDM systems. When the channel is a slowly varying one, a MIMO-IOFDM system can be used, instead of estimating the channel over multiple OFDM blocks, so that, an increase

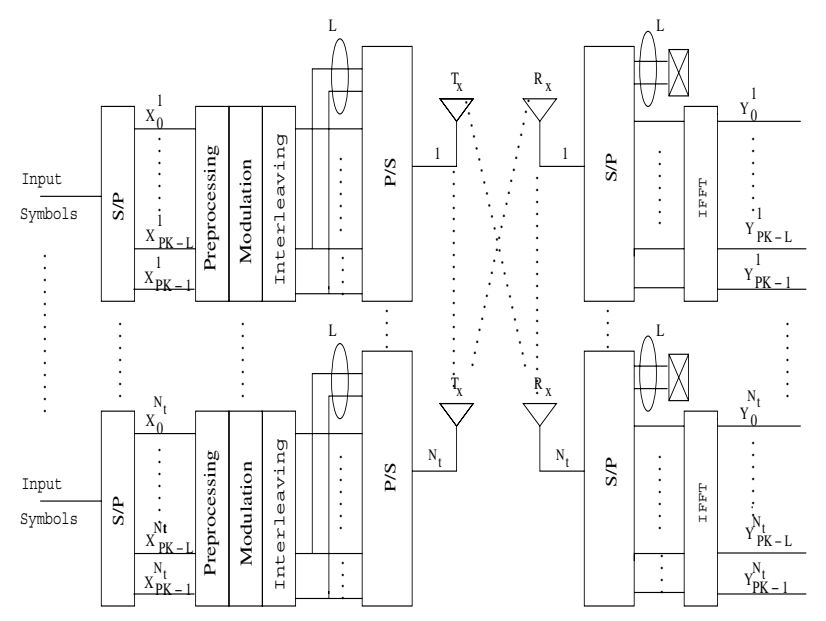

Fig. 1. Model for MIMO-IOFDM

in the code-rate can be achieved even during the channel estimation period.

\section{SYSTEM MODEL}

The system under consideration is depicted in Figure(1). For the analysis, we use the matrix model for IOFDM proposed in [3], developed based on the discussions in [5]. At each transmit antenna, the data is first preprocessed by a diagonal matrix (as described in [1]), and then modulated by an IFFT, and then interleaved. Then a cyclic prefix of length $\nu \geq L-1$, where $L$ is the maximum length of all the channels. Given $N_{t}$ transmit antennas, the received block at the $q^{t h}$ receive antenna after removing the cyclic prefix is given by:

$$
\underline{\mathbf{y}}^{q}(n)=\sum_{p=1}^{N_{t}} \mathbf{H}_{c i r c}^{q, p}(n) \mathbf{L}_{P K} \mathbf{C}_{P K}^{H} \mathbf{G} \underline{\mathbf{X}}^{p}(n)+\underline{\eta}^{q}(n)
$$

where, $\mathbf{H}_{\text {circ }}^{\mathbf{q}, \mathbf{p}}$ is a circulant matrix, of dimension $P K \times P K$, the first column being given by, $\left[\begin{array}{lll}\underline{\mathbf{h}}^{q, p^{T}} & (n) & \underline{0}_{1 \times(P K-L)}\end{array}\right]^{T}$, where $\underline{\mathbf{h}}^{q, p}=\left[h_{0}{ }^{q, p}(n) \ldots h_{L-1}{ }^{q, p}(n)\right]^{T}$ is the channel impulse response between the $p^{t h}$ transmit antenna and the $q^{t h}$ 
receive antenna. $\underline{\mathbf{X}}^{p}=\left[X_{0}^{p}(n) \ldots X_{0}^{p}(n)\right]^{T}$, is the transmitted vector from the $p^{t h}$ transmit antenna at time index $n$ and $K$ is the number of subcarriers in the system and $P$ is the number of data blocks in the IOFDM system. $\mathbf{L}_{P K}$ is a $P K \times P K$ interleaving matrix, $\mathbf{C}_{P K}$ is a $P K \times P K$ block diagonal matrix, with each of the $K \times K$ block diagonal matrix being a $K$ point DFT matrix. $\mathbf{G}$ is a diagonal preprocessing matrix of size $P K \times P K$ as described in [1]. $\eta^{q}$ is the AWGN vector. Taking the $P K$ point FFT of eqn. $(\overline{1})$, we have,

$$
\begin{aligned}
\underline{\mathbf{Y}}^{q}(n)= & \sum_{p=1}^{N_{t}} \mathbf{D}^{q, p}(n) \mathbf{F}_{P K} \mathbf{L}_{P K} \mathbf{C}_{P K}^{H} \mathbf{G} \underline{\mathbf{X}}^{p}(n) \\
& +\underline{\mathbf{G}}^{q}(n)
\end{aligned}
$$

where, $\mathbf{D}^{q, p}$ is a diagonal matrix with the frequency response of $\underline{\mathbf{h}}^{q, p}$ as it's diagonal elements, and $\underline{\Xi}^{q}$ is the frequency domain noise, and $\mathbf{F}_{P K}$ is a $P K$ point DFT matrix.

\section{CHANNEL ESTIMATION}

In this section, our channel estimation scheme is derived. Let $\underline{\mathbf{X}}^{p}(n)=\underline{\mathbf{S}}^{p}(n)+\underline{\mathbf{B}}^{p}(n)$, where, $\underline{\mathbf{S}}^{p}(n)$ is some arbitrary $P K \times 1$ data vector and $\underline{\mathbf{B}}^{p}(n)$ is some arbitrary $P K \times 1$ training sequence(TS) vector. Now, from eqn.2, we obtain,

$$
\begin{aligned}
\underline{\mathbf{Y}}^{q}(n)= & \sum_{p=1}^{N_{t}} \operatorname{diag}\left(\mathbf{F}_{P K} \mathbf{L}_{P K} \mathbf{C}_{P K}^{H} \mathbf{G} \underline{\mathbf{S}}^{p}(n)\right. \\
& \left.+\mathbf{F}_{P K} \mathbf{L}_{P K} \mathbf{C}_{P K}^{H} \mathbf{G} \underline{\mathbf{B}}^{p}(n)\right) \mathbf{F}_{P K}^{0: L-1} \underline{\mathbf{h}}^{q, p}(n) \\
& +\underline{\mathbf{\Xi}}^{q}(n)
\end{aligned}
$$

where, $\mathbf{F}_{P K}^{0: L-1}$ is the first $L$ columns of $\mathbf{F}_{P K}$.

\subsection{Properties of the product $\mathbf{F}_{P K} \mathbf{L}_{P K} \mathbf{C}_{P K}^{H} \mathbf{G}$ :}

1) From [6], we have, $\mathbf{F}_{P K} \mathbf{L}_{P K} \mathbf{C}_{P K}^{H} \mathbf{G}=\mathbf{L}_{P K}^{H} \mathbf{C}_{K P} \mathbf{L}_{P K}$, where, $\mathbf{C}_{K P}$ is a $P K \times P K$ block diagonal matrix with each of the $P \times P$ block diagonal matrix being a $P$ point DFT matrix. The product is also a symmetric matrix.

2) Consider a $P=3$ system, then, $\mathbf{L}_{P K}^{H} \mathbf{C}_{K P} \mathbf{L}_{P K}$ which is nothing but a permuted block DFT matrix, has the following structure:

$$
\left[\begin{array}{ccc}
\mathbf{U} & \mathbf{U} & \mathbf{U} \\
\mathbf{U} & \mathbf{V} & \mathbf{W} \\
\mathbf{U} & \mathbf{W} & \mathbf{V}
\end{array}\right]
$$

where, each of $\mathbf{U}, \mathbf{V}, \mathbf{W}$ are $K \times K$ scaled identity matrices. Consider a $3 K \times 1$ vector $\underline{\mathbf{a}}=\left[\begin{array}{ll}\underline{\mathbf{a}}_{1}^{T} & \underline{\mathbf{a}}_{2}^{T} \\ \mathbf{a}_{3}^{T}\end{array}\right]^{T}$ where, each of the $\underline{\mathbf{a}}_{i}$ is a $K \times 1$ vector. The term $\mathbf{L}_{P K}^{H} \mathbf{C}_{K P} \mathbf{L}_{P K} \underline{\mathbf{a}}$ for $P=3$ can be written as : $\sum_{i=1}^{3} \mathbf{M}_{i}\left[\begin{array}{lll}\underline{\mathbf{a}}_{i}^{T} & \mathbf{a}_{i}^{T} & \mathbf{a}_{i}^{T}\end{array}\right]^{T}$, where, $\mathbf{M}_{\mathbf{i}}$ is the block diagonal matrix, whose blocks are from the $i^{t h}$ column of $\mathbf{L}_{P K}^{H} \mathbf{C}_{\mathbf{K P}} \mathbf{L}_{\mathbf{P K}}$, i.e., $\mathbf{M}_{2}$ is equal to blockdiagonal $(\mathbf{U}, \mathbf{V}, \mathbf{W})$, etc., $\mathbf{M}_{i}$ is a $P K \times P K$ matrix. These properties can be extended to any $\mathrm{P}$ in general.

In equation(3), we will now have the equality given by,

$\mathbf{F}_{P K} \mathbf{L}_{P K} \mathbf{C}_{P K}^{H} \mathbf{G}_{\mathbf{B}^{p}}(n)=\sum_{i=1}^{P} \mathbf{M}_{i}\left[\underline{\mathbf{B}}_{i}^{p^{T}}(n) \ldots \underline{\mathbf{B}}_{i}^{p^{T}}(n)\right]^{T}$ where $\underline{\mathbf{B}}^{p}(n)=\left[\underline{\mathbf{B}}_{1}^{p^{T}}(n) \ldots \underline{\mathbf{B}}_{P}^{p^{T}}(n)\right]^{T}$ and $\underline{\mathbf{B}}_{i}^{p}(n)$ is a $K \times 1$ vector from $\underline{\mathbf{B}}^{p}(n)$. Also,

$$
\begin{aligned}
& \sum_{p=1}^{N_{t}} \operatorname{diag}\left(\mathbf{F}_{P K} \mathbf{L}_{P K} \mathbf{C}_{P K}^{H} \mathbf{G}^{\mathbf{B}^{p}}(n)\right) \mathbf{F}_{P K}^{0: L-1} \underline{\mathbf{h}}^{q, p}(n)= \\
& \sum_{p=1}^{N_{t}} \operatorname{diag}(\sum_{i=1}^{P} \mathbf{M}_{i} \underbrace{\underbrace{\mathbf{B}_{i}^{T}}_{i}(n) \ldots \underline{\mathbf{B}}_{i}^{p^{T}}(n)]}_{P K \times 1 \text { vector }}{ }^{T}) \mathbf{F}_{P K}^{0: L-1} \underline{\mathbf{h}}^{q, p}(n)
\end{aligned}
$$

Define $\mathbf{B}_{i}^{p}=\operatorname{diag}\left(\left[\underline{\mathbf{B}}_{i}^{p^{T}}(n) \ldots \underline{\mathbf{B}}_{i}^{p^{T}}(n)\right]\right)$, for $i=1, \ldots, P$ and $p=1, \ldots, N_{t}$. If $N_{t}=1$, then L.H.S. in eqn.4 is $\left[\mathbf{M}_{1} \mathbf{B}_{1}^{1} \mathbf{F}_{P K}^{0: L-1} \cdots \mathbf{M}_{P} \mathbf{B}_{P}^{1} \mathbf{F}_{P K}^{0: L-1}\right]\left[\underline{\mathbf{h}}^{q, 1^{T}}(n) \ldots \underline{\mathbf{h}}^{q, 1^{T}}(n)\right]^{T}$. It can be seen that it is sufficient for tones in one of the $P$ data blocks in an IOFDM system to contain all the power, the number of tones in that block being equal to $T / P$, where $T \geq N_{t} L$ assuming $T$ to be a factor of $P$. This is true for all the transmit antennas, i.e., tones in only one of the $\mathrm{P}$ data blocks will contain all the power associated for training in each of the transmit antennas. Let this be an $i^{\text {th }}$ block in each of the transmit antenna, define $\mathbf{B}_{\text {pow }}^{p}=\mathbf{M}_{i} \mathbf{B}_{i}^{p}$, $p=1, \ldots, N_{t}$ The eqn. 3 can now be rewritten as,

$$
\begin{aligned}
\underline{\mathbf{Y}}^{q}(n) & = \\
& \sum_{p=1}^{N_{t}} \operatorname{diag}\left(\mathbf{F}_{P K} \mathbf{L}_{P K} \mathbf{C}_{P K}^{H} \mathbf{G} \underline{\mathbf{S}}^{p}(n)\right) \mathbf{F}_{P K}^{0: L-1} \underline{\mathbf{h}}^{q, p}(n) \\
& +\sum_{p=1}^{N_{t}} \mathbf{B}_{p o w}^{p} \mathbf{F}_{P K}^{0: L-1} \underline{\mathbf{h}}^{q, p}(n)+\underline{\boldsymbol{\Xi}}^{q}(n)
\end{aligned}
$$

Define $\mathbf{A}=\left[\mathbf{B}_{\text {pow }}^{1} \mathbf{F}_{P K}^{0: L-1} \cdots \mathbf{B}_{p o w}^{N_{t}} \mathbf{F}_{P K}^{0: L-1}\right]$. Assuming full column rank for $\mathrm{A}$,

$$
\begin{aligned}
& \mathbf{A}^{\dagger} \underline{\mathbf{Y}}^{q}(n)= \\
& \quad \mathbf{A}^{\dagger} \sum_{p=1}^{N_{t}} \operatorname{diag}\left(\mathbf{F}_{P K} \mathbf{L}_{P K} \mathbf{C}_{P K}^{H} \mathbf{G} \underline{\mathbf{S}}^{p}(n)\right) \mathbf{F}_{P K}^{0: L-1} \underline{\mathbf{h}}^{q, p}(n) \\
& \quad+\underline{\mathbf{h}}^{q}(n)+\mathbf{A}^{\dagger} \underline{\boldsymbol{\Xi}}^{q}(n)
\end{aligned}
$$

where, $\underline{\mathbf{h}}^{q}(n)=\left[\underline{\mathbf{h}}^{q, 1^{T}}(n) \cdots \underline{\mathbf{h}}^{q, N_{t}{ }^{T}}(n)\right]^{T}$ If the term given by $\mathbf{A}^{\dagger} \sum_{p=1}^{N_{t}} \operatorname{diag}\left(\mathbf{F}_{P K} \mathbf{L}_{P K} \overline{\mathbf{C}}_{P K}^{H} \mathbf{G} \underline{\mathbf{S}}^{p}(n)\right) \mathbf{F}_{P K}^{0: L-1} \underline{\mathbf{h}}^{q, p}(n)$ should be equal to zero, for $p=1, \ldots, N_{t}$, then, it is possible only if the pilot tones and the data occupy disjoint positions within an IOFDM block, just as the case in [2]. This results in,

$$
\begin{gathered}
\underline{\hat{\mathbf{h}}}^{q}(n)=\tilde{\mathbf{A}}^{\dagger} \underline{\tilde{\mathbf{Y}}}^{q}(n) \\
\underline{\hat{\mathbf{h}}}^{q}(n)=\underline{\mathbf{h}}^{q}(n)+\tilde{\mathbf{A}}^{\dagger} \underline{\tilde{\Xi}}^{q}(n)
\end{gathered}
$$


where, the term $\tilde{\mathbf{A}}=\left[\tilde{\mathbf{B}}_{\text {pow }}^{1} \tilde{\mathbf{F}}_{P K}^{0: L-1} \cdots \tilde{\mathbf{B}}_{\text {pow }}^{N_{t}} \tilde{\mathbf{F}}_{P K}^{0: L-1}\right]$, the term, $\tilde{\mathbf{B}}_{\text {pow }}^{p}=\operatorname{diag}\left(\right.$ non zero entries of $\left.\mathbf{B}_{\text {pow }}^{p}\right), \tilde{\mathbf{F}}_{P K}^{0: L-1}$ , $\underline{\mathbf{Y}}^{q}(n)$ and $\tilde{\boldsymbol{\Xi}}^{q}(n)$ are the corresponding rows of $\mathbf{F}_{P K}^{0: L-1}$, $\underline{\mathbf{Y}}^{q}(n)$ and $\underline{\boldsymbol{\Xi}}^{q}(n)$ respectively.

\section{OPTIMAL TRAINING SEQUENCE DESIGN}

The channel estimation analysis is evidently identical to the channel estimation analysis in section $I V$ in reference [2].

In this section, optimum training sequences for channel estimation in MIMO-IOFDM systems is derived. Let $T$ be the number of pilot tones required for training in each of the transmit antennas. The necessary condition for identifiability is, $T \geq L N_{t}$.

On similar lines to that described in [2], the condition for the mean square error of the channel estimates to be minimum is that $\tilde{\mathbf{A}}^{H} \tilde{\mathbf{A}}$ should be a diagonal matrix. For this to be true, we need,

1) The non-zero entries in each of $\tilde{\mathbf{B}}_{\text {pow }}^{p}, p=1, \ldots, N_{t}$ to be equispaced.

2) The $(k, k)^{t h}$ entry of the product $\tilde{\mathbf{B}}_{\text {pow }}^{u^{H}} \tilde{\mathbf{B}}_{\text {pow }}^{v}$ is denoted by $\left[\tilde{\mathbf{B}}_{\text {pow }}^{u^{H}} \tilde{\mathbf{B}}_{\text {pow }}^{v}\right]_{k, k}$. As shown in [2], the condition given by, $\sum_{k=1}^{T}\left[\tilde{\mathbf{B}}_{\text {pow }}^{u^{H}} \tilde{\mathbf{B}}_{\text {pow }}^{v}\right]_{k, k} e^{-j \frac{2 \pi}{T} k \phi}=0$, has to be satisfied $\forall u, v \in\left\{1, \ldots, N_{t}\right\}$, and, $\forall \phi \in\{-L+1, \ldots, L-1\}$.

The design of the pilot tones has to be done taking care of the above two criteria. The criterion are satisfied by designing the tones such that,

$$
\left[\tilde{\mathbf{B}}_{\text {pow }}^{u^{H}} \tilde{\mathbf{B}}_{\text {pow }}^{v}\right]_{k, k}=e^{-j \frac{2 \pi}{T} n_{u v} k}
$$

where, $\left[\tilde{\mathbf{B}}_{p o w}^{u}\right]_{k, k}=e^{-j \frac{2 \pi}{T} n_{u} k}$ and $n_{u v}=n_{u}-n_{v}$ with $n_{u, v} \in Z$. As discussed in [2], the second criterion is satisfied if $\frac{n_{u, v}+\phi}{T} \notin Z \forall \phi$ and $\forall u, v \in\left\{1, \ldots, N_{t}\right\}$ with $u \neq v$. One of the choices for design would be with $n_{u}=$ $(u-1) L, \forall u \in\left\{1, \ldots, N_{t}\right\}$. Now we have a design for the matrix $\tilde{\mathbf{B}}_{\text {pow }}^{p}$, which is obtained by allotting power to the tones in the $i^{t h}$ data block in the $p^{t h}$ transmit antenna. Now we have to design the $T / P$ tones in that data block in which the tones are allotted power, so that the required $\tilde{\mathbf{B}}_{\text {pow }}^{p}$ is obtained. This can be done as follows : let the numbering of the $P$ blocks in an IOFDM block be done as $[0, P-1, \ldots, 1]$. Then, the block in which the tones receive the power is given by, $i_{\text {pow }}=\operatorname{P\operatorname {mod}}((u-1) L)$, $\forall u \in\left\{1, \ldots, N_{t}\right\}$. The $T / P$ tones which are present in the $i_{\text {pow }}^{t h}$ block can be designed as follows : define $\underline{\mathbf{\mathbf { B }}}_{i_{\text {pow }}}^{u}$ as the $(T / P) \times 1$ vector of tones present in the $i_{\text {pow }}^{\text {th }}$ block, transmitted from the $u^{\text {th }}$ transmit antenna, then

$$
\left[\underline{\tilde{\mathbf{B}}}_{i_{\text {pow }}^{u}}^{u}\right]_{k}=\left(P^{\frac{1}{2}}\right) *\left[\tilde{\mathbf{B}}_{\text {pow }}^{u}\right]_{k, k}
$$

$\forall k=1, \ldots, T / P$ and $\forall u \in\left\{1, \ldots, N_{t}\right\}$. Also, these $T / P$ tones will have to be equispaced and equipowered(assuming
Table 1. Comparison of Optimal Training Sequence(TS) Requirements for MIMO-OFDM and MIMO-IOFDM

\begin{tabular}{|c|c|}
\hline System & TS requirement \\
\hline MIMO- & Equipowered + Equispaced [6] \\
OFDM & + Phase-shift Orthogonality [2] \\
\hline MIMO- & Equispaced + Tones in one of the $P$ \\
IOFDM & data blocks should be equipowered + \\
& $\begin{array}{c}\text { Tones in corresponding positions of remaining } \\
P-1 \text { blocks should be equal to zero. }\end{array}$ \\
\hline
\end{tabular}

constant modulus sequences) within the $i_{\text {pow }}^{\text {th }}$ block of data. The tones in the corresponding positions in the rest of the data blocks should be equal to zero. This completes the design of the optimal pilot tones for MIMO-IOFDM system. A comparison between the training sequence requirements for MIMO-OFDM (as designed in [2]) and MIMO-IOFDM systems is as shown in Table(1).

It is also necessary that the design of the pilot tones needs to be as discussed above. This fact can be understood by comparing the equations for an OFDM system with $P * K$ subcarriers and an IOFDM system with $P$ data blocks and $K$ subcarriers. The receiver equations for the two systems are, respectively,

$$
\underline{\mathbf{Y}}^{q}(n)=\sum_{p=1}^{N_{t}} \mathbf{D}^{q, p}(n) \underline{\mathbf{X}}_{O F D M}^{p}(n)+\underline{\Xi}^{q}(n)
$$

as in [2], and, using property(1) of Section(3.1) in eqn.(2),

$$
\begin{aligned}
\underline{\mathbf{Y}}^{q}(n)= & \sum_{p=1}^{N_{t}} \mathbf{D}^{q, p}(n) \mathbf{L}_{P K}^{H} \mathbf{C}_{K P} \mathbf{L}_{P K} \underline{\mathbf{X}}_{I O F D M}^{p}(n) \\
& +\underline{\boldsymbol{\Xi}}^{q}(n)
\end{aligned}
$$

where, $\underline{\mathbf{X}}_{O F D M}^{p}(n)$ and $\underline{\mathbf{X}}_{I O F D M}^{p}(n)$ are the input vectors in OFDM and IOFDM systems, respectively. Now, the design of $\underline{\mathbf{X}}_{p}^{O F D M}(n)$ for MMSE of the channel estimates has been discussed in [2]. Hence, the design for an IOFDM system should match the vector $\underline{\mathbf{X}}_{p}^{\text {IOFDM }}(n)$ such that the two designs are equivalent, in the sense that, $\underline{\mathbf{X}}_{I O F D M}^{p}(n)=\left(\mathbf{L}_{P K}^{H} \mathbf{C}_{K P} \mathbf{L}_{P K}\right)^{-1} \underline{\mathbf{X}}_{O F D M}^{p}(n)$ for the design of the IOFDM vector to be optimal. It can be shown that this argument also results in unique set of input tone vectors which match our design for the IOFDM system.

\section{SIMULATIONS AND DISCUSSIONS}

In the simulations, an AWGN channel with 8-taps is used. The number of subcarriers used, $K=64$ and value of $P$ used is 4 . A cyclic prefix of 8 symbols is added once for every $P$ blocks being transmitted in order to avoid IBI. 2 transmit and 4 receive antennas were used. The matrix 


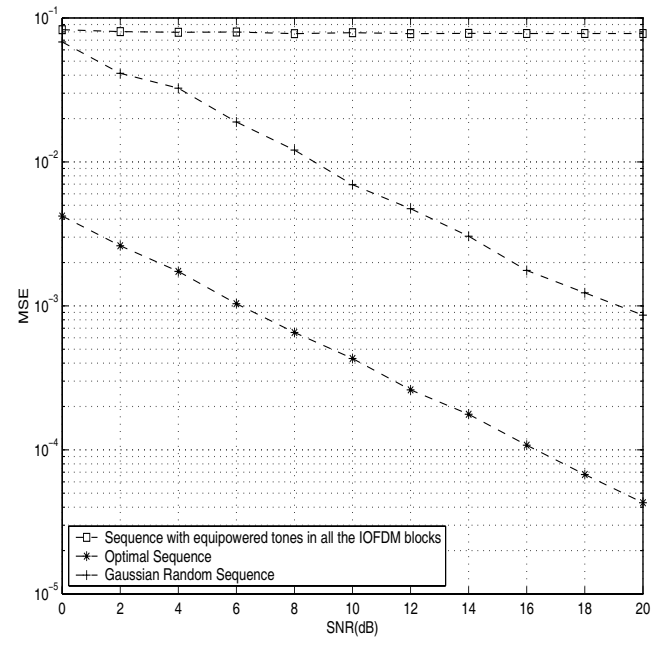

Fig. 2. Comparison of MSE in $\underline{\mathbf{h}}^{1}$ vs SNR for MIMOIOFDM System for different training sequences. $\left(P=4, K=64, L=8\right.$, Modulation:BPSK, $N_{t}=2$ )

model for IOFDM has been used in our simulations. In order to compare the optimal training sequence design for IOFDM with that of the design in [2], we have simulated both the methods of estimating the channel over 4 consecutive OFDM blocks and with the IOFDM system with $P=4$.

As shown in figure(2), which depicts the variation of channel MSE versus the SNR, using the training sequence designed in section(4) for MIMO-IOFDM outperforms the use of random sequences and tones with power in all the data blocks in the system. The random training sequence has been used in [2] to compare with the optimal training sequence design. The sequence with tones being equipowered in all the data blocks consists of equispaced tones $\left(L * N_{t}=\right.$ $8 * 2=16$ tones) in an IOFDM block, with the design as in [2]. Further, Table(2) shows us that the two schemes of estimating the channel when the channel is slowly varying, one of them in which estimation is done over multiple OFDM blocks (as discussed in [2]) and the other is by using IOFDM system instead, result in identical performance as far as the channel MSE is concerned.

\section{CONCLUSIONS}

In this paper, a channel estimation scheme based on pilot tones for MIMO-IOFDM system is proposed. It is found that, in order to minimize the MSE of the channel estimates in the training stage, the pilot tones must be equispaced in frequency, equipowered, and that the entire power has to be allotted to the pilot tones in one of the $P$ blocks in each transmit antenna, with the tones in the corresponding positions in the other blocks being equal to zero. Simulation results show that the proposed training sequence design out-
Table 2. Comparison of MSE of Channel Estimates for MIMO-IOFDM ( $\left.P=4, K=64, N_{t}=2, L=8\right)$ and MIMO-OFDM ( $\left.K=64, N_{t}=2, L=8\right)$, the channel being estimated over 4 consecutive OFDM blocks (as in [2]).

\begin{tabular}{|c|c|c|}
\hline SNR(dB) & $\begin{array}{c}\text { MSE } \\
\text { MIMO-OFDM }\end{array}$ & $\begin{array}{c}\text { MSE } \\
\text { MIMO-IOFDM }\end{array}$ \\
\hline 0 & $4.3 \times 10^{-3}$ & $4.2 \times 10^{-3}$ \\
2 & $2.6 \times 10^{-3}$ & $2.6 \times 10^{-3}$ \\
4 & $1.7 \times 10^{-3}$ & $1.7 \times 10^{-3}$ \\
6 & $1.1 \times 10^{-3}$ & $1.0 \times 10^{-3}$ \\
8 & $7.0 \times 10^{-4}$ & $7.0 \times 10^{-4}$ \\
10 & $4.0 \times 10^{-4}$ & $4.0 \times 10^{-4}$ \\
12 & $3.0 \times 10^{-4}$ & $3.0 \times 10^{-4}$ \\
14 & $2.0 \times 10^{-4}$ & $2.0 \times 10^{-4}$ \\
\hline
\end{tabular}

performs the other training sequences with respect to the MSE of the channel estimates. The Table(2) shows that when the channel is varying slowly, MIMO-IOFDM system can used instead of the scheme in [2], since it increases the code rate of the system relative to the scheme in [2].

\section{REFERENCES}

[1] V. G. S. Prasad and K. V. S. Hari, "Interleaved Orthogonal Frequency Division Multiplexing System", in the Proc. IEEE ICASSP, Orlando, Florida, pp. III.2745 - 2748, May 2002. An elaborate version to appear also in IEEE Trans. on Signal Processing

[2] I. Barhumi, G. Leus and M. Moonen, "Optimal training sequences for channel estimation in MIMO OFDM systems in mobile wireless channels," in Int. Zurich Symp. on Broadband Communications, pp.44-1 - 44-6, Feb. 2002.

[3] Karthik Subramanian, K. V. S. Hari, "Alternative Interleaving Schemes for Interleaved Orthogonal Frequency Division Multiplexing," in Proc. IEEE TENCON, Bangalore, India, Oct. 2003.

[4] V. G. S. Prasad and K. V. S. Hari, "Space-Time Block Coded Interleaved Orthogonal Frequency Division Multiplexing System," in Proc. ICPWC 2002, New Delhi, India, Dec. 2002. An elaborate version to appear in Book Chapter, Springer Verlag, 2003.

[5] Zhengdao Wang, Georgios B. Giannakis, "Wireless Multicarrier Communications -where Fourier meets Shannon," IEEE Signal Processing Magazine, pp. 29-48, May 2000.

[6] J. H. Manton, "Optimal Training Sequences and Pilot Tones for OFDM Systems", IEEE Communication Letters, Vol. 5, No. 4, April 2001. 